Cahiers $d u$ MONDE RUSSE

\section{Cahiers du monde russe}

Russie - Empire russe - Union soviétique et États indépendants

$46 / 4 \mid 2005$

L'invention d'une politique humanitaire

\title{
Francine Hirsch, Empire of Nations
}

\author{
Juliette Cadiot
}

\section{OpenEdition \\ Journals}

Édition électronique

URL : https://journals.openedition.org/monderusse/6651

DOI : 10.4000/monderusse.6651

ISSN : $1777-5388$

Éditeur

Éditions de l'EHESS

Édition imprimée

Date de publication : 1 décembre 2005

Pagination : 954-956

ISBN : 2-7132-2057-2

ISSN : $1252-6576$

\section{Référence électronique}

Juliette Cadiot, "Francine Hirsch, Empire of Nations », Cahiers du monde russe [En ligne], 46/4 | 2005, mis en ligne le 29 juin 2009, consulté le 03 septembre 2022. URL : http://journals.openedition.org/ monderusse/6651 ; DOI : https://doi.org/10.4000/monderusse.6651

Ce document a été généré automatiquement le 3 septembre 2022.

Tous droits réservés 


\title{
Francine Hirsch, Empire of Nations
}

\author{
Juliette Cadiot
}

\section{RÉFÉRENCE}

Francine HIRSCH, Empire of Nations. Ethnographic Knowledge and the Making of

the Soviet Union. Ithaca : Cornell University Press, 2005, XIII-367p.

1 Issu de sa thèse, le livre de Francine Hirsch analyse la construction de l'URSS à travers l'étude des formes de représentations, notamment statistiques et ethnographiques, de la diversité nationale et des politiques de «territorialisation» des nationalités. Cette approche par le biais des travaux des ethnographes et des recensements permet à l'auteur de caractériser l'expérience soviétique comme un processus complexe d'élaboration d'un "empire des nations». S'inscrivant dans un tournant historiographique qui a radicalement rompu depuis les années 1990 avec une tradition qui avait fait des leaders bolcheviks des «briseurs de nations", l'auteur tend à prouver que ces derniers ont au contraire entrepris une politique volontariste visant leur construction. Hirsch, s'appuyant sur les travaux désormais classiques de Benedict Anderson ${ }^{1}$, étudie comment certains outils - recensement, musées et cartes ethnographiques - ont été mis en URSS au service de l'organisation d'un État fondé sur l'institutionnalisation des différences nationales entre 1917 et la Seconde Guerre mondiale. Partant du constat que beaucoup de Soviétiques, au moment de la révolution, ne connaissaient pas leur «nationalité », elle décrit les mesures prises par l'État central pour aboutir à une double assimilation: chaque citoyen fut sommé de se reconnaître à la fois comme Soviétique et comme membre d'une nationalité particulière. Hirsch explique ces politiques par l'impératif de modernisation du nouveau régime. En liant la population à des nationalités, voire à des nations pourvues d'un territoire, d'une culture et du droit de les défendre, en les arrachant à des identifications plus locales, les dirigeants bolcheviks auraient voulu susciter un évolutionnisme impulsé par l'État (a state sponsored evolutionism).

2 Le premier chapitre présente un groupe d'ethnographes, formés avant la révolution et bientôt réunis dans l'Académie des sciences, qui jouera un rôle particulièrement 
important dans les premières années de l'Union soviétique. Dirigés par l'orientaliste Sergej Ol'denburg, leader du parti cadet et membre du Gouvernement Provisoire, ces ethnographes se mirent au service de l'État bolchevik, notamment en lui proposant de poursuivre leurs travaux de recensement et de cartographie ethnique du territoire. Cette alliance entre les anciennes élites savantes de l'empire et le nouveau régime est emblématique. Le projet de reconstruction de l'État à l'aide des outils offerts par la science était partagé par les anciens adversaires politiques et les scientifiques mirent très largement leur expertise au service du nouveau pouvoir. Les ethnographes en particulier prirent part au débat concernant la territorialisation soviétique et fournirent les données cartographiques et statistiques nécessaires à son dessin.

3 Hirsch relate dans le second chapitre les débats sur la régionalisation: devait-elle être fondée sur des impératifs de rationalité économique ou sur le respect des frontières nationales? Les diverses formes envisagées renvoyaient non seulement à la compétition entre grands organes étatiques (commissariat aux Nationalités, Gosplan), mais aussi à la volonté de redéfinir les rapports de domination issus de la période précédente. Une discussion sur l'éventualité d'une colonisation à la soviétique, non impérialiste mais modernisatrice, fut menée non seulement par les administrateurs, mais aussi par les ethnographes conviés à statuer sur ces problèmes, notamment quand il s'agissait de populations considérées comme "arriérées». L'étude du recensement général de la population de 1926 (chapitre III) permet à l'auteur d'envisager la dimension proprement identitaire du projet de construction nationale soviétique. Les hésitations d'une partie de la population pour s'attribuer une nationalité et les difficultés des ethnographes pour établir une liste exhaustive des nationalités comportant des ethnonymes appropriés permettent d'avoir une idée de l'extrême complexité des identifications ethniques.

Dans le quatrième chapitre, Hirsch étudie le découpage des frontières nationales, qui a été fondé en partie sur les statistiques, mais a également mis en présence différents acteurs centraux et locaux et a abouti à divers compromis. Ainsi, les habitants d'une localité ont pu lancer une pétition pour exiger que leur village (ou qu'un terrain) soit attribué à une république donnée, mais, ce faisant, ils utilisaient et s'appropriaient les catégories nationales établies par l'État. L'auteur propose une typologie des diverses modalités de détermination des frontières entre les différentes républiques dans les années 1920 et les relie à des formes d'identification nationale, offrant ainsi une vision dynamique de ces processus.

5 Ensuite Hirsch s'intéresse aux travaux des ethnographes, à l'organisation d'expositions présentant les peuples de l'URSS dans leur spécificité, puis dans leur modernité, soviétique. Elle décrit les effets de la soviétisation de la discipline ethnographique, qui fut radicalement remise en cause, au sein de l'Académie des sciences. Le chapitre suivant, inédit par rapport à la thèse, traite des efforts entrepris par les savants soviétiques pour répondre à la science allemande sur la base d'un refus du concept de race biologique. Ce positionnement scientifique des anthropologues et des biologistes soviétiques leur fut imposé par le pouvoir central. Mais les années 1930 débouchèrent aussi sur la Grande Terreur et les déportations en fonction de l'appartenance nationale, désormais inscrite sur le passeport non plus en fonction de l'autodéfinition, mais de l'origine ethnique. Au cours des recensements de 1937 et 1939, le nombre de nationalités reconnues fut réduit de manière drastique, les divers "petits peuples" étant rassemblés dans des unités « nationales » globalisantes. Enfin, une hiérarchie entre les nations fut établie. 
6 La lecture de l'ouvrage est agréable, l'écriture dynamique et la complexité des phénomènes étudiés clairement énoncée. Néanmoins, les partis pris méthodologiques de l'auteur ne sont pas toujours suffisamment explicités ni exploités. Hirsch a tendance à surestimer la position des ethnographes au sein de l'administration soviétique, car il n'est pas sûr qu'ils aient eu un réel impact sur les décisions politiques. Traiter en parallèle le discours de Lenin et de Stalin et celui des ethnographes paraît peu approprié. Il aurait été possible, plus modestement, de mieux valoriser le fait qu'étudier les ethnographes, et surtout leurs archives, permet de restituer une réalité sociale et ethnique complexe et mouvante, peu visible à travers des textes plus théoriques ou des archives au contenu plus politique. Les ethnographes et les statisticiens en particulier se trouvaient au moment des recensements en rapport direct avec la population et devaient élaborer des catégories recouvrant des identifications multiples et changeantes. Ils pouvaient occasionnellement être les interlocuteurs de pouvoirs locaux soucieux d'être représentés au centre comme ils le désiraient. Dès lors, c'est du fait de leur position d'interface entre divers acteurs sociaux dont on entend généralement peu la voix, notamment celle des simples citoyens soviétiques, que les ethnographes sont intéressants à étudier. En se focalisant sur les discours scientifiques mis au service du politique, l'auteur offre parfois une vision trop rationnelle et dirigiste de l'État. Les mesures entreprises pour aboutir à la création d'identités nationales paraissent artificielles et sans fondement social préalable. L'auteur évoque à peine les mouvements nationaux ainsi que les événements qui ont secoué l'empire de Russie entre 1905 et 1917, période au cours de laquelle la question des périphéries, des nationalismes locaux, de la fédération fut en discussion et où s'opéra une véritable "nationalisation » du discours politique et de la société. Les nouveaux citoyens soviétiques paraissent dès lors avoir été soumis à des catégories arbitraires, artificielles, décidées par un pouvoir tout-puissant, issu de la coalition de la science et de la politique. Or, les archives du recensement de 1926 montrent certes qu'une partie de la population a eu du mal à préciser sa nationalité, préférant se définir à l'aide d'ethnonymes locaux ou généraux, mais qu'elle était toutefois capable de se positionner dans une communauté ; par ailleurs, des statistiques très précises sur la composition ethnique de l'URSS ont finalement pu être produites parce que les habitants ont globalement su répondre à la question.

7 Le livre de Francine Hirsch est stimulant, riche en informations, notamment sur les constructions identitaires ou encore sur le caractère colonial de l'expérience soviétique. Il constitue enfin un essai particulièrement sophistiqué d'application des nouvelles tendances historiographiques.

\section{NOTES}

1. B. Anderson, L'imaginaire national : réflexions sur l'origine et l'essor du nationalisme, Paris : La Découverte, 1996 (éd. orig.: Imagined Communities: Reflections on the Origin and Spread of Nationalism, Londres : Verso, 1983). 